
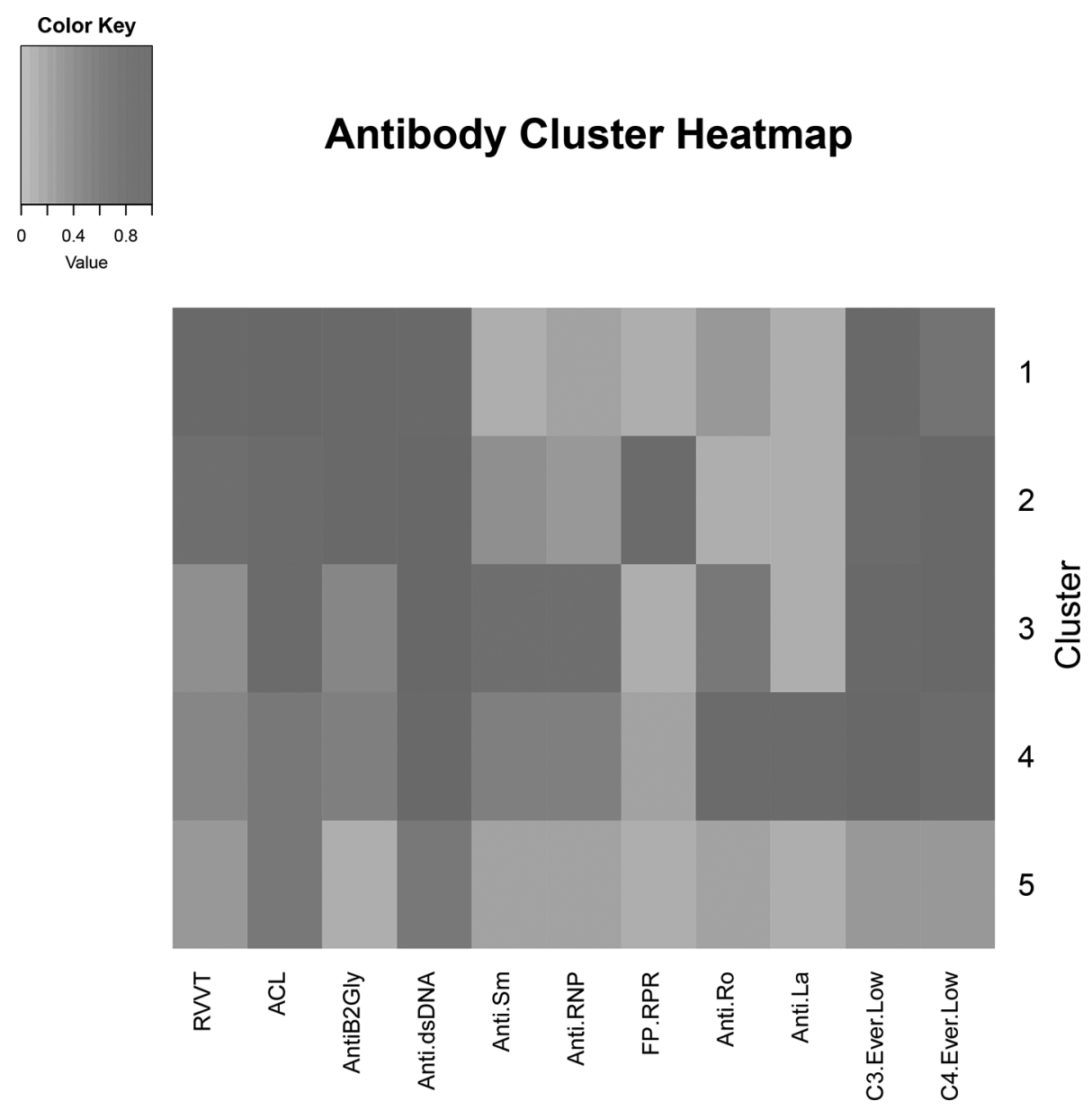

\title{
Autoantibodies
}

Abstract 103 Figure 1 Heatmap clustering. Heatmap shows the frequency of each autoantibody by cluster: russell viper venom test (RVVT), anticardiolipin (ACL), anti-beta2 glycoprotein I (AntiB2Gly), antidsDNA, antiSm, antiRNP, false positive (FP)RPR, antiRo, antiLa, C3 ever low, and C4 ever low. Antibody frequency is colored from grey $(0.0$, low) to red $(1.0$, high). Heatmap rows corresponding to clusters $1,2,3,4$, and 5 are indicated.

Results There were five unique clusters. Clusters 1 and 2 had high frequencies of hypocomplementemia, antiphospholipid antibodies, and anti-dsDNA. Cluster 2 additionally had a high frequency of FP-RPR. Cluster 3 had high frequencies of hypocomplementemia, anticardiolipin, anti-Sm, anti-RNP, and antidsDNA. Cluster 4 had high frequencies of hypocomplementemia, anti-Ro, anti-La, and anti-dsDNA. Cluster 5 had only moderate frequencies of anti-dsDNA and anti-cardiolipin antibody. Two clusters were defined by antiphospholipid antibodies. Two clusters were defined by anti-RNP and anti-Sm. Multiple clusters had elevated anti-dsDNA and hypocomplementemia.

Conclusions PH in SLE is defined by five unique autoantibody clusters. Antiphospholipid and anti-RNP/Sm clusters clearly separate, which likely indicates different pathophysiologic mechanisms. Four of the five clusters have antidsDNA and low complement components. Our next step is to exploreboth in the literature and within our cohortthe natural history and response to treatment of these clusters.

Funding Source(s): NIH Grant R01-AR069572

Clustering of Autoantibodies in Lupus-Associated Pulmonary Hypertension

\section{PREDICTORS OF READMISSION IN LUPUS COMPARED TO GENERAL MEDICARE PATIENTS}

${ }^{1}$ Christie Bartels*, ${ }^{2}$ Ann Chodara, ${ }^{2}$ Xing Wang, ${ }^{2}$ Fangfang Shi, ${ }^{2}$ Amy Kind. ${ }^{1}$ University of Wisconsin School of Medicine and Public Health; ${ }^{2}$ UW School of Medicine and Public Health

\subsection{6/lupus-2019-Ism. 104}

Background Medicare measures readmissions within 30 days of hospitalization as a marker of care quality across several conditions, although beyond hospital quality, neighborhood disadvantage is a known predictor of readmission. While not a reporting condition, a 2010 study noting 27\% lupus readmissions led to our interest in examining readmission in lupus versus general Medicare including the impact of neighborhood disadvantage.

Methods Using a 20\% US national Medicare sample, we identified all patients with inpatient hospitalizations 2013-14 to compare odds of 30 day readmission among patients with SLE to the general Medicare population. Inclusion required age >18 y.o., 12 mos. Medicare Part A/B (30d. post discharge), and being alive at discharge. Covariates included age, gender, race, ethnicity, prior Medicaid, comorbidity, prior hospitalization, length of stay, hospital volume, medical school 


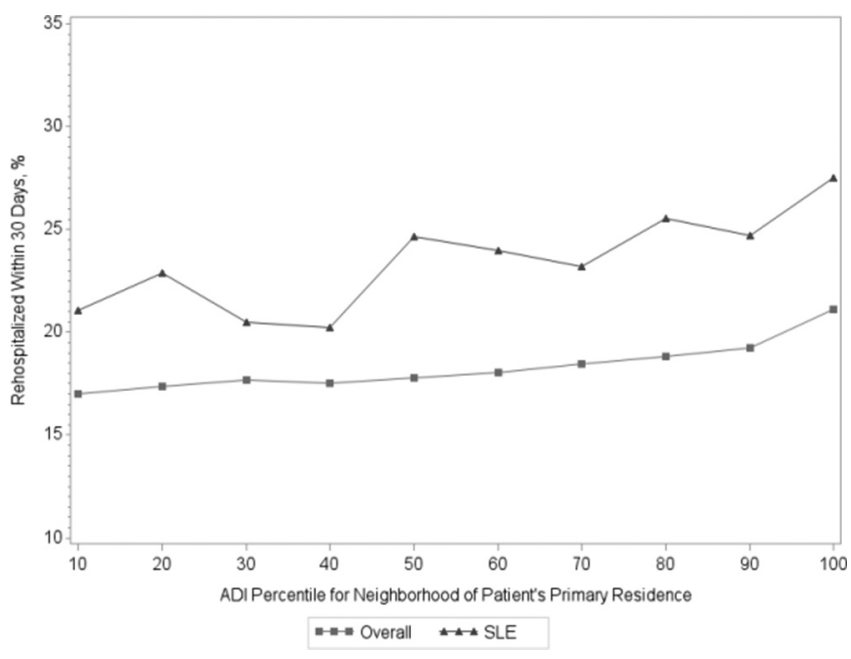

Abstract 104 Figure 1 30-day readmission by neighborhood disadvantage (ADI decile)

affiliation, rural urban commuter area, and area deprivation index (ADI)a measure of neighborhood disadvantage derived from patients census block group (www.neighborhoodatlas. medicine.wisc.edu). Analysis used multivariable logistic regression clustered by patient.

Results Nearly 1.4 million acute Medicare hospitalizations were analyzed including 10868 lupus hospitalizations. Patients with lupus were younger $(55 \%$ of hospitalizations in 18 $<65$ y.o.) and $89 \%$ were female. Patients with lupus were over twice as likely to be Black or Hispanic (29\% vs $12 \%$; $5 \%$ vs $2 \%$ respectively). Among lupus patients, $46 \%$ had history of Medicaid, 65\% were Medicare eligible due to disability, and $15 \%$ were eligible due to end stage renal disease (ESRD), compared to 30\% Medicaid, 32\% disability, and 3\% ESRD among general Medicare.

Lupus readmissions ranged from 20\% within lower disadvantage to $27 \%$ within the greatest decile of ADI neighborhood disadvantage, compared to $17 \%-21 \%$ in general Medicare regardless of ADI decile (Figure 1). Before adjustment, patients with SLE were $60 \%$ more likely to be readmitted than non-lupus Medicare patients (Unadj. OR 1.61 (1.51, 1.71)). Lupus remained a readmission risk in adjusted models
(Adj. OR $1.28(1.20,1.36))$. SLE patients with 30 day readmissions were more likely to be $<65$ y.o., Black, to have had prior Medicaid or hospitalization, and other comorbidities; significant interactions were seen between ADI quintile and Medicaid predicting readmission.

Conclusions Among nationally representative Medicare hospitalizations, having lupus was a major readmission risk factor. Hospitalized patients with lupus who lived in a neighborhood with greatest disadvantage had higher 30 day readmissions, impacting nearly 1 in 3 patients. Future studies are needed to examine readmission causes and interventions to systematically reduce readmissions among vulnerable hospitalized patients with lupus.

Funding Source(s): None

\section{PROVIDER PERCEPTIONS ON THE MANAGEMENT OF LUPUS DURING PREGNANCY: BARRIERS TO IMPROVED CARE}

Megan EB Clowse*, Jessica Revels, Amanda M Eudy, Gillian Schmidler-Sanders, Laura Neil, Lisa G Criscione-Schreiber. Duke University

\subsection{6/lupus-2019-Ism.105}

Background More than half of pregnancies in women with systemic lupus erythematosus (lupus) result in adverse outcomes for both mother and fetus. As demonstrated in the PROMISSE study, pregnancies managed by experts can have outcomes similar to the general population. However, many women with lupus do not receive this level of care and suffer complications at higher rates. We sought to identify aspects of current community rheumatologic care that could be improved to decrease the frequency of poor lupus pregnancy outcomes. Methods Focus groups with clinical rheumatologists were held at state rheumatology conferences in North Carolina, South Carolina, Virginia, and Northern California. The interview guide was based on the PRECEDE/PROCEED framework, seeking factors that predisposed, reinforced, and enabled physician practice patterns. Focus groups were recorded, transcribed, and coded to identify the elements of this framework. A group of women with lupus on their reproductive journey contributed to our understanding of the dilemmas and care provided.

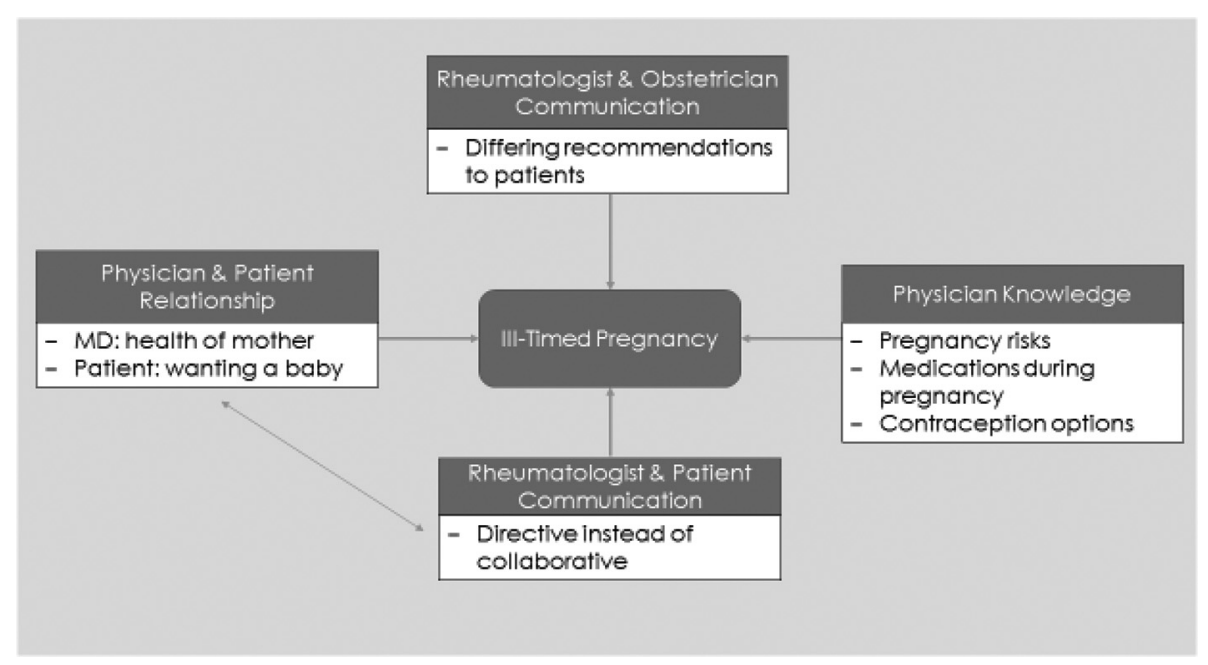

Abstract 105 Figure 1 Model of modifiable contributors to ill-timed lupus pregnancy 